\title{
A General Conceptual Framework for Decoherence in Closed and Open Systems
}

\author{
Mario Castagnino $^{\mathrm{a}}$ - Roberto Laura ${ }^{\mathrm{b}}$ - Olimpia Lombardi ${ }^{\mathrm{c}}$ \\ ${ }^{a}$ CONICET-IAFE, Universidad Nacional de Buenos Aires. \\ b IFIR-Universidad Nacional de Rosario. \\ c CONICET-Universidad Nacional de Buenos Aires. Corresponding author: olimpiafilo@arnet.com.ar
}

\begin{abstract}
In this paper we argue that the formalisms for decoherence originally devised to deal just with closed or open systems can be subsumed under a general conceptual framework, in such a way that they cooperate in the understanding of the same physical phenomenon. This new perspective dissolves certain conceptual difficulties of the einselection program but, at the same time, shows that the openness of the quantum system is not the essential ingredient for decoherence.
\end{abstract}

\section{Introduction}

At present, the environment-induced decoherence (EID) approach is considered a "new orthodoxy" in the physicists community (Bub 1997); it has been fruitfully applied in many areas of physics and supplies the basis of new technological developments. In the philosophy of physics, EID has been viewed as a relevant element for the interpretation of quantum mechanics (Bacciagaluppi and Hemmo 1994, 1996) and for the explanation of the emergence of classicality from the quantum world (Elby 1994; Healey 1995).

The great success of EID has given rise to the idea that decoherence necessarily requires the interaction between an open quantum system and an environment of many, potentially infinite, degrees of freedom. However, the historical roots of the decoherence program can be found in certain attempts to explain the emergence of classicality in closed systems. In turn, at present other approaches have been proposed, and in several of them the openness of the system is not an essential factor. These new approaches are usually conceived as rival to EID or even as dealing with different physical phenomena.

The aim of this paper is to argue that this is not the case; on the contrary, formalisms originally devised to deal just with closed or open systems can be subsumed under a general 
conceptual framework and viewed as complementary perspectives for understanding the same physical phenomenon. This new viewpoint dissolves certain conceptual difficulties of the EID program but, at the same time, shows that the openness of the quantum system is not the essential ingredient for decoherence, as commonly claimed.

\section{The Historical Development of the Decoherence Program}

From a historical perspective, the decoherence program finds its origin -thought, of course, not under this name- in the attempts to explain how a coherent pure state becomes a final decohered mixture with no interference terms. Three general periods can be identified in the development of this program:

First period: closed systems. In the fifties and the early sixties, some authors directed their attention to the emergence of classical macroscopic features from quantum microscopic descriptions (van Kampen 1954; van Hove 1957, 1959; Daneri et. al 1962). In this period, the issue was treated in the context of the study of irreversibility and, therefore, closed systems were considered. On this basis, the states indistinguishable from the viewpoint of certain "gross" observables were described by the same coarse-grained state, whose evolution was proved to reach equilibrium in a certain relaxation time. The main problem of this period was that the relaxation times so obtained turned out to be too long to account for experimental results (see Omnes 2005).

Second period: open systems. In the seventies, the emergence of classicality begun to be conceived in terms of the quantum measurement which, in turn, was addressed from an opensystem perspective (Zeh 1970, 1971, 1973). On the basis of these precedents, the EID approach was systematized and developed mainly by Zurek in a great number of works (Zurek 1981, 1991, 2003; Paz and Zurek 2002). In this context, an open system is considered in interaction with its environment, and the evolution of its reduced state is studied; EID proves that, in many physical models, the interference terms of the reduced state rapidly vanish and the system decoheres in an extremely short decoherence time. This result solves the main problem of the first period; however, the foundations of the EID program are still threatened by certain conceptual problems derived from its open-system perspective (we will return on this point in the next section). 
Third period: open and closed systems. Although 'EID' is still considered almost as a synonym for 'decoherence', in the last times other approaches have been proposed to face the conceptual difficulties of EID (Diosi 1987, 1989; Milburn 1991; Casati and Chirikov 1995a, 1995b; Adler 2004). Some of these accounts are clearly non-dissipative (Bonifacio et al. 2000; Ford and O’Connell 2001; Frasca 2003; Sicardi Schifino et al. 2003), that is, not based on the dissipation of energy from the system to the environment. Among them, the self-induced decoherence (SID) approach shows that a closed quantum system with continuous spectrum may decohere by destructive interference (see the works of Castagnino’s group in the references).

In spite of the fact that, at present, formalisms for open and closed systems coexist, in the literature both kinds of approaches are often presented as alternative scenarios for decoherence, and even as theories dealing with different physical phenomena (Schlosshauer 2005). In the next sections we will challenge this common view by showing that EID and SID can be understood in the context of a general conceptual framework.

\section{The Conceptual Problems of EID}

In spite of the great success of EID, this approach still has to face two conceptual difficulties.

\section{3.a) The “Open-System” Problem}

According to EID, decoherence is a consequence of the interaction between an open system and its environment; this process is what "einselects" the quantum states that become the candidates to classical states. Therefore, decoherence must always be accompanied by other manifestations of openness, such as the dissipation of energy into the environment. Zurek even considers that the prejudice which seriously delayed the solution of the problem of the emergence of classicality is itself rooted in the fact that the role of the openness of the system was traditionally ignored (Paz and Zurek 2002; Zurek 2003).

If only open systems may decohere, from this perspective the issue of the emergence of classicality in closed systems, in particular, in the Universe as a whole, cannot even be posed. Zurek expresses the criticism to EID in the following terms: "the Universe as a whole is still a single entity with no 'outside' environment, and, therefore, any resolution involving its division into systems is unacceptable” $(1994,181)$. This objection, pointed out only by few authors (Pessoa Jr. 
1998), has led to the development of the non-dissipative approaches to decoherence which, for this reason, are usually viewed as alternative or rival to the EID approach.

\section{3.b) The "Defining Systems" Problem}

When EID is applied to cosmology, the strategy always consists in splitting the Universe into some degrees of freedom representing the system, and the remaining degrees of freedom that are supposed to be non accessible and, therefore, play the role of the environment; the same strategy is followed in the case of "internal" environments, such as collections of phonons or other internal excitations. The possibility of "internal" environments shows that EID supplies no general criterion for distinguishing between the system and its environment: the partition of the whole closed system is decided case by case, and usually depends on the previous assumption of the observables that will behave classically (see discussion in Castagnino and Lombardi 2004).

The absence of a general criterion for deciding where to place the "cut" between system and environment is a difficulty particularly serious for an approach that insists on the essential role played by the openness of the system in the emergence of classicality. Zurek recognizes this problem as a shortcoming of his proposal: "In particular, one issue which has been often taken for granted is looming big as a foundation of the whole decoherence program. It is the question of what are the 'systems' which play such a crucial role in all the discussions of the emergent classicality. This issue was raised earlier, but the progress to date has been slow at best" (Zurek 1998, 122).

As we will see, these problems, which seem to be serious conceptual obstacles for the EID approach, loose their original strength when decoherence is understood from a new general perspective.

\section{A General Conceptual Framework for Decoherence}

As emphasized by Omnes (2001, 2002), decoherence is a particular case of the phenomenon of irreversibility in quantum mechanics, whose explanation can be sketched in the following terms. Since the quantum state $\rho(t)$ of a closed system follows a unitary evolution, it cannot reach a final equilibrium state for $t \rightarrow \infty$. Therefore, if the non-unitary evolution towards equilibrium is to be accounted for, a set $\mathcal{O}$ of relevant observables $O_{\mathrm{R}}$ has to be selected, and a coarse-grained state 
$\rho_{\mathrm{G}}(t)$ has to be defined, such that $\left\langle O_{\mathrm{R}}\right\rangle_{\rho(t)}=\left\langle O_{\mathrm{R}}\right\rangle_{\rho_{\mathrm{G}}(t)}$ for any $O_{\mathrm{R}} \in \mathcal{O}$ (see Castagnino et al. 2005). It is $\rho_{\mathrm{G}}(t)$ (or, equivalently, $\left\langle O_{\mathrm{R}}\right\rangle_{\rho(t)}$ ) what reaches a final equilibrium value through its non-unitary evolution.

Since decoherence is an irreversible process, it should be understood in the context of the general account of irreversibility on the basis of three general steps:

Step 1: The set $\mathcal{O}$ of relevant observables is defined. In fact, all the different approaches to decoherence select a set of relevant observables in terms of which the time behavior of the system is described: gross observables (van Kampen), macroscopic observables of the apparatus (Daneri), relevant observables (Omnes), observables of the open system (EID), van Hove observables (SID), etc.

Step 2: The expectation value $\left\langle O_{\mathrm{R}}\right\rangle_{\rho(t)}$, for any $O_{\mathrm{R}} \in \mathcal{O}$, is obtained. This step can be performed in two different but equivalent ways:

$*\left\langle O_{\mathrm{R}}\right\rangle_{\rho(t)}$ is directly computed as the expectation value of $O_{\mathrm{R}}$ in the unitarily evolving state $\rho(t)$.

* The coarse-grained state $\rho_{\mathrm{G}}(t)$, such that $\left\langle O_{\mathrm{R}}\right\rangle_{\rho(t)}=\left\langle O_{\mathrm{R}}\right\rangle_{\rho_{\mathrm{G}}(t)}$ for any $O_{\mathrm{R}} \in \mathcal{O}$, is defined, and its non-unitary evolution (governed by a master equation) is computed.

Step 3: It is proved that $\left\langle O_{\mathrm{R}}\right\rangle_{\rho(t)}=\left\langle O_{\mathrm{R}}\right\rangle_{\rho_{\mathrm{G}}(t)}$ reaches a final value $\left\langle O_{\mathrm{R}}\right\rangle_{\rho_{*}}$ :

$$
\lim _{t \rightarrow \infty}\left\langle O_{\mathrm{R}}\right\rangle_{\rho(t)}=\lim _{t \rightarrow \infty}\left\langle O_{\mathrm{R}}\right\rangle_{\rho_{\mathrm{G}}(t)}=\left\langle O_{\mathrm{R}}\right\rangle_{\rho *}
$$

Therefore, $\rho_{\mathrm{G}}(t)$ evolves towards a final stable state $\rho_{\mathrm{G}^{*}}$ that is obviously diagonal in its own eigenbasis (see Castagnino et al. 2006). But the unitarily evolving state $\rho(t)$ has only a weak limit:

$$
W-\lim _{t \rightarrow \infty} \rho(t)=\rho_{*}
$$


This means that, although the off-diagonal terms of $\rho(t)$ never vanish through the unitary evolution, the system decoheres from the observational point of view given by any relevant observable $O_{\mathrm{R}} \in \mathcal{O}$.

In the next subsections, we will argue for the generality of this conceptual framework by applying it to the SID and the EID approaches. This will show that, in spite of the fact that SID deals with closed systems and EID describes open systems, the general concept of decoherence expressed by Steps 1 to 3 lies behind both approaches.

\section{4.a) SID: Decoherence in Closed Systems}

In the SID approach, the three steps are explicit in the formalism. For conciseness, here we will present SID in the simplest case (see references for more general models).

Step 1: Let us consider a closed system $S$ endowed with a Hamiltonian $H$ with continuous spectrum $\omega \in[0, \infty): H|\omega\rangle=\omega|\omega\rangle$. The relevant observables $O_{\mathrm{R}}$ belong to the van Hove space $\mathcal{O}_{\text {SID }}$, whose basis is $\left.\left.\{\mid \omega), \mid \omega, \omega^{\prime}\right)\right\}$, where $\left.\mid \omega\right)=|\omega\rangle\langle\omega|$ and $\left.\mid \omega, \omega^{\prime}\right)=|\omega\rangle\left\langle\omega^{\prime}\right|$ :

$$
\left.\left.O_{\mathrm{R}}=\int_{0}^{\infty} O(\omega) \mid \omega\right) d \omega+\int_{0}^{\infty} \int_{0}^{\infty} O\left(\omega, \omega^{\prime}\right) \mid \omega, \omega^{\prime}\right) d \omega d \omega^{\prime}
$$

States $\rho$ are represented by linear functionals on $\mathcal{O}_{\text {SID }}$, that is, they belong to the dual space $\mathcal{O}_{\text {SID }}^{\prime}$ with basis $\left\{\left(\omega \mid,\left(\omega, \omega^{\prime} \mid\right\}\right.\right.$, the cobasis of $\left.\left.\{\mid \omega), \mid \omega, \omega^{\prime}\right)\right\}$ :

$$
\rho=\int_{0}^{\infty} \rho(\omega)\left(\omega \mid d \omega+\int_{0}^{\infty} \int_{0}^{\infty} \rho\left(\omega, \omega^{\prime}\right)\left(\omega, \omega^{\prime} \mid d \omega d \omega^{\prime}\right.\right.
$$

If states satisfy the usual requirements ( $\rho(\omega)$ real and positive and $\int_{0}^{\infty} \rho(\omega) d \omega=1$ ), they belong to a convex set $\mathcal{S} \subset \mathcal{O}^{\prime}{ }_{\text {SID }}$.

Step 2: The expectation value of the observable $O_{\mathrm{R}} \in \mathcal{O}_{\text {SID }}$ in the state $\rho(t) \in \mathcal{S}$ can be computed as the action of the functional $\rho(t)$ on the operator $O_{\mathrm{R}}$ :

$$
\left\langle O_{\mathrm{R}}\right\rangle_{\rho(t)}=\int_{0}^{\infty} \rho^{*}(\omega) O(\omega) d \omega+\int_{0}^{\infty} \int_{0}^{\infty} \rho^{*}\left(\omega, \omega^{\prime}\right) O\left(\omega, \omega^{\prime}\right) e^{i \frac{\omega-\omega^{\prime}}{\hbar}} d \omega d \omega^{\prime}
$$


Step 3: Since the $O_{\mathrm{R}} \in \mathcal{O}_{\mathrm{SID}}$ are defined in such a way that the function $\rho^{*}\left(\omega, \omega^{\prime}\right) O\left(\omega, \omega^{\prime}\right)$ be regular (precisely, $\mathbb{L}_{1}$ in variable $\nu=\omega-\omega^{\prime}$ ), the Riemann-Lebesgue theorem can be applied to eq.(5). As a consequence, the second term of this equation vanishes:

$$
\lim _{t \rightarrow \infty}\left\langle O_{\mathrm{R}}\right\rangle_{\rho(t)}=\int_{0}^{\infty} \rho^{*}(\omega) O(\omega) d \omega
$$

This means that, for $t \rightarrow \infty$, the expectation value of any $O_{\mathrm{R}} \in \mathcal{O}_{\text {SID }}$ can be computed as if the

system were in a final state $\rho_{*}=\int_{0}^{\infty} \rho(\omega)(\omega \mid d \omega$ that has only diagonal terms in the eigenbasis of the Hamiltonian:

$$
\lim _{t \rightarrow \infty}\left\langle O_{\mathrm{R}}\right\rangle_{\rho(t)}=\left\langle O_{\mathrm{R}}\right\rangle_{\rho_{*}}
$$

This result can also be expressed as a weak limit:

$$
W-\lim _{t \rightarrow \infty} \rho(t)=\rho_{*}
$$

Summing up, through Steps 1 to 3 (see the coincidence between eqs.(7)-(8) and eqs.(1)-(2)), SID cancels interference and leads to a final diagonal state $\rho_{*}$. Although SID strictly applies in the continuous case, it also leads to approximate decoherence in quasi-continuous models, that is, discrete models where (i) the energy spectrum has a small discrete energy spacing, and (ii) the functions of energy are such that the sums in which they are involved can be approximated by Riemann integrals.

\section{4.b) EID: Decoherence in Open Systems}

In the case of the EID approach, Steps 1 to 3 are usually not explicit. However, this approach can be rephrased in the context of the new general framework.

Step 1: Let us consider a closed system $U$ that can be decomposed into a proper system $S$ and an environment $E$, whose Hilbert spaces are $\mathcal{H}=\mathcal{H}_{\mathrm{S}} \otimes \mathcal{H}_{\mathrm{E}}, \mathcal{H}_{\mathrm{S}}$ and $\mathcal{H}_{\mathrm{E}}$. The von NeumannLiouville space of $U$ is $\mathcal{L}=\mathcal{H} \otimes \mathcal{H}=\mathcal{L}_{\mathrm{S}} \otimes \mathcal{L}_{\mathrm{E}}$, where $\mathcal{L}_{\mathrm{S}}=\mathcal{H}_{\mathrm{S}} \otimes \mathcal{H}_{\mathrm{S}}$ and $\mathcal{L}_{\mathrm{E}}=\mathcal{H}_{\mathrm{E}} \otimes \mathcal{H}_{\mathrm{E}}$. A generic observable reads:

$$
O=O_{\mathrm{S}} \otimes O_{\mathrm{E}} \in \mathcal{L}, \quad \text { with } O_{\mathrm{S}} \in \mathcal{L}_{\mathrm{S}} \text { and } O_{\mathrm{E}} \in \mathcal{L}_{\mathrm{E}}
$$


In this case, the relevant observables $O_{\mathrm{R}} \in \mathcal{O}_{\mathrm{EID}} \subset \mathcal{L}$ are those corresponding to the proper system $S$ :

$$
O_{\mathrm{R}}=O_{\mathrm{S}} \otimes I_{\mathrm{E}} \in \mathcal{O}_{\mathrm{EID}}
$$

where $I_{\mathrm{E}}$ is the identity operator in $\mathcal{L}_{\mathrm{E}}$.

Step 2: The expectation value of the observable $O_{\mathrm{R}} \in \mathcal{O}_{\mathrm{EID}}$ in the state $\rho$ of $U$ reads:

$$
\left\langle O_{\mathrm{R}}\right\rangle_{\rho}=\operatorname{Tr}\left(\rho O_{\mathrm{R}}\right)=\operatorname{Tr}\left(\rho\left(O_{\mathrm{S}} \otimes I_{\mathrm{E}}\right)\right)
$$

But when we define the reduced density operator $\rho_{\mathrm{s}}$ of $S$ by tracing over the environmental degrees of freedom, $\rho_{\mathrm{S}}=\operatorname{Tr}_{\mathrm{E}} \rho$, that expectation value can also be obtained as:

$$
\left\langle O_{\mathrm{R}}\right\rangle_{\rho(t)}=\operatorname{Tr}\left(\rho(t) O_{\mathrm{R}}\right)=\operatorname{Tr}\left(\rho(t)\left(O_{\mathrm{S}} \otimes I_{\mathrm{E}}\right)\right)=\left\langle O_{\mathrm{S}}\right\rangle_{\rho_{\mathrm{S}}(t)}
$$

Step 3: The EID approach studies the evolution of $\rho_{\mathrm{S}}(t)$ governed by a generalized master equation; for many physical models it shows that, for $t \rightarrow \infty, \rho_{\mathrm{S}}(t)$ reaches a final stable state $\rho_{\mathrm{S}^{*}}$, which is obviously diagonal in its own eigenbasis. But if we remember the definition of $\rho_{\mathrm{S}}$ as a partial trace, we can obtain the limit of the expectation values of eq.(12) as:

$$
\lim _{t \rightarrow \infty}\left\langle O_{\mathrm{R}}\right\rangle_{\rho(t)}=\lim _{t \rightarrow \infty}\left\langle O_{\mathrm{S}}\right\rangle_{\rho_{\mathrm{S}}(t)}=\left\langle O_{\mathrm{S}}\right\rangle_{\rho_{\mathrm{S}^{*}}}=\left\langle O_{\mathrm{R}}\right\rangle_{\rho_{*}}
$$

Therefore, for any observable $O_{\mathrm{R}} \in \mathcal{O}_{\text {EID }}$ :

$$
\lim _{t \rightarrow \infty}\left\langle O_{\mathrm{R}}\right\rangle_{\rho(t)}=\left\langle O_{\mathrm{R}}\right\rangle_{\rho_{*}}
$$

This result can also be expressed as a weak limit:

$$
W-\lim _{t \rightarrow \infty} \rho(t)=\rho_{*}
$$

If the just obtained eqs.(14)-(15) are compared with the corresponding eqs.(7)-(8) of the SID approach and with eqs.(1)-(2), the similarity among them can be easily verified. This shows that the EID approach can also be formulated in terms of the closed composite system $U$ and, from this perspective, it can be explained in the context of the new general framework just proposed. 


\section{Closed and Open Systems}

If decoherence in closed and open systems can be subsumed under a common conceptual framework, the corresponding formalisms are not alternative, as usually claimed, but complementary: both cooperate in the understanding of the same physical phenomenon. Furthermore, this new general perspective allows us to explore the relationship between the decoherence of a closed system and the decoherence of any of its subsystems. In fact, it is not difficult to prove that, given a closed system $U$ partitioned into two subsystems $S_{1}$ and $S_{2}$, if $U$ decoheres according to SID, then $S_{1}$ and $S_{2}$ decohere according to EID for their respective relevant observables, and this fact does not depend on the particular partition selected (see Castagnino et al. 2006). This means that, when the whole composite system decoheres, the subsystems will also decohere no matter how many degrees of freedom they have. This conclusion shows that it is not always necessary for the decoherence of an open system its interaction with an environment with many, potentially infinite, degrees of freedom: the decoherence of the whole composite system imposes a physical situation as strong as to lead to the decoherence of any of its subsystems.

If there is a physical relationship between the decoherence of a closed system and the decoherence of its subsystems, there must be also a meaningful relationship between the respective decoherence times $t_{\mathrm{DU}}$ and $t_{\mathrm{DS}}$. When these times are computed by means of the SID formalism, it can be proved that (Castagnino and Lombardi 2005; Castagnino et al. 2006): (i) if the interaction between the system $S$ and its environment $E$ is macroscopic, $t_{\mathrm{DS}} \approx 10^{-39} \mathrm{~s}$, and (ii) if the interactions of the parts of $E$ between themselves are microscopic, $t_{\mathrm{DU}} \approx 10^{-15} \mathrm{~s}$. As expected, $t_{\mathrm{DS}} \ll t_{\mathrm{DU}}$ : in general, the time that the whole system needs to decohere is much longer than the decoherence time of a small subsystem strongly coupled with the rest of the degrees of freedom. This result reasonably agrees with those obtained by EID, where $t_{\mathrm{DS}}$ is also extremely short, provided that both times are so close to the Plank time that only have a qualitative meaning.

These conclusions allow us to clarify certain points that may remain obscure when the models are studied with numerical techniques but with no conceptual perspective. In a recent paper, Schlosshauer (2005) studies a simple bath model where a single spin-1/2 particle interacts with an environment consisting of a collection of $N$ non-interacting spin-1/2 particles. According to EID (Zurek 1982), the single particle decoheres in interaction with the environment. But when 
Schlosshauer applies the SID formalism to the model and tests the results by means of numerical simulations, he does not obtain decoherence for the whole system. On this basis, the author concludes that the destructive interference introduced by the Riemann-Lebesgue theorem, conceptual basis of the SID, is not always efficient: SID would not account for a case of decoherence that has been perfectly explained by EID. However, when the model is studied from a conceptual viewpoint, the conclusions are completely different. As we have seen, the decoherence of the whole composite system implies the decoherence of any of its subsystems; therefore, when any subsystem does not decohere, we can guarantee that the composite system will neither decohere. This is precisely the case of the spin-bath model, where the environment cannot decohere to the extent that its component particles are uncoupled to each other. This result is completely natural from a physical point of view: a collection of free evolving particles is unable to reach a final decohered state. Therefore, in spite of the fact that one subsystem decoheres, the whole system will not decohere because the other subsystem is endowed with a trivial Hamiltonian. This shows that certain results, which may seem puzzling when only considered by means of numerical simulations, turn out to be necessary conceptual consequences of the full understanding of the physical phenomenon.

\section{Dissolving the Conceptual Problems of EID}

When decoherence is understood in the new general framework, the conceptual difficulties of the EID program turn out to be not as serious as originally supposed. In fact:

a) Closed quantum systems may decohere, and their decoherence times can be computed. Furthermore, in spite of the fact that EID focuses on open systems, it can also be formulated from the perspective of the composite system and, in this case, meaningful relationships between the behavior of the whole system and the behavior of its subsystems can be explained.

b) The "defining systems" problem is simply dissolved by the fact that the splitting of the closed system into an open subsystem and an environment is just a way of selecting the relevant observables of the closed system. Since there are many different sets of relevant observables depending on the observational viewpoint adopted, the same closed system can be decomposed in many different ways: each decomposition represents a decision about which degrees of freedom are relevant and which can be disregarded in any case. If there is no privileged or "essential" 
decomposition, there is no need of an unequivocal criterion for deciding where to place the cut between "the” system and "the” environment.

Although the new framework neutralizes the conceptual difficulties of the EID approach, also points to some warnings about the way in which the proposal is usually presented. From the new perspective, the insistence on the essential role played by the openness of a system and its interaction with the environment in the phenomenon of decoherence sounds rather misleading. The essential physical fact is that, among all the observational viewpoints that may be adopted to study a closed system, some of them determine a subset of relevant observables for which the system decoheres.

\section{Coarse-grainings and Projections}

As we have seen, when the unitarily evolving state $\rho(t)$ weakly tends to $\rho_{*}$, the coarse-grained state $\rho_{\mathrm{G}}(t)$, such that $\left\langle O_{\mathrm{R}}\right\rangle_{\rho(t)}=\left\langle O_{\mathrm{R}}\right\rangle_{\rho_{\mathrm{G}}(t)}$ for any $O_{\mathrm{R}} \in \mathcal{O}$, evolves towards a final stable state $\rho_{\mathrm{G}^{*}}$. As it is well known, coarse-graining amounts to a projection that reduces the number of components of a state vector (Mackey 1989). In our case, it can be proved that $\rho_{\mathrm{G}}(t)$ is the result of the projection of $\rho(t)$ onto the space $\mathcal{O}$ of relevant observables, and the same relationship holds between $\rho_{\mathrm{G}^{*}}$ and $\rho_{*}$; as a consequence, for $t \rightarrow \infty$ the coarse-grained state $\rho_{\mathrm{G}}(t)$ tends to $\rho_{\mathrm{G}^{*}}$ not only in a weak sense, but also in a strong sense (Castagnino et al. 2006):

$$
\lim _{t \rightarrow \infty} \rho_{\mathrm{G}}(t)=\rho_{\mathrm{G}^{*}}
$$

This means that what approaches to a final stable state in the infinite time limit is a coarse-grained magnitude defined by the relevant observables. In other words, decoherence is a coarse-grained process, resulting from the coarse-graining introduced by the relevant observables on the underlying unitary evolution. It turns out to be clear that this conclusion is valid both for open and for closed systems: like any other irreversible evolution, decoherence is a process that only arises in a coarsegrained level of description, but that cannot be accounted for in the underlying quantum level with its unitary evolutions.

This conclusion has to be taken into account particularly when the results of decoherence are applied to face the problems of interpretation in quantum mechanics. In some cases, decoherence is 
used to explain the definite value of certain observables as if it were a quantum evolution described at the same level as the unitary evolutions of the quantum states. But when the coarse-grained nature of decoherence is recognized, one has to be very careful in appealing to it to solve interpretational problems. Perhaps to think that the interpretation of quantum mechanics will supply a relevant meaning to the results of decoherence is a better strategy than to search in decoherence the solutions for interpretation.

Finally, somebody could argue that, if decoherence is a coarse-grained process, classicality is a subjective appearance only due to our limited access to reality (Zeh 1971; Stamp 1995; d'Espagnat 1995). We do not endorse this conclusion because we reject the subjective interpretation of coarse-

graining. For us, the fact that two descriptions are related by means of a projection does not imply that only one of them is objective, and the other is irremediably subjective. From an ontologically pluralist perspective, different ontologies may coexist, each one of them with its corresponding objective description: the relationship of projection between two different descriptions does not diminish the objectivity of one of them. Of course, these brief remarks do not amount to a full argument (for a detailed discussion, see Lombardi 2002; Lombardi and Labarca 2005): the careful treatment of this matter requires a discussion that largely exceeds the purposes of the present paper.

\section{Conclusions}

In this paper we have presented a general conceptual framework that encompasses both EID and SID, and probably other decoherence approaches. From this perspective, the difficulties that threaten the EID program can be viewed under a new light that mitigates their conceptual relevance. In turn, when it is accepted that the formalisms of decoherence for open and closed systems are not rival or alternative, but that they cooperate in the understanding of the same physical reality, the results obtained in both cases turn out to be relevant: for instance, the large amount of experimental confirmations of EID (see Joos et al. 2003), the complete description of the classical limit of quantum mechanics (Castagnino 2004; Castagnino and Gadella 2006; Castagnino and Lombardi 2003, 2006b) and the study of the role of complexity in decoherence (Castagnino 2005, 2006; Castagnino and Lombardi 2006a) in the case of SID, and the compatibility in the magnitude order of the decoherence times computed by EID and SID (Castagnino and Lombardi 2005) can be all retained as important acquisitions in the new general framework. 


\section{References}

Adler, Stephen (2004), Quantum Theory as an Emergent Phenomenon. Cambridge: Cambridge University Press.

Bonifacio, Rodolfo, Stefano Olivares, Paolo Tombesi and David Vitali (2000), "Model-Independent Approach to Nondissipative Decoherence”, Physical Review A 61: 053802.

Bub, Jeffrey (1997), Interpreting the Quantum World. Cambridge: Cambridge University Press.

Bacciagaluppi, Guido and Meir Hemmo (1994), “Making Sense of Approximate Decoherence”, Proceedings of the Philosophy of Science Association 1: 345-354.

-_- (1996), “Modal Interpretations, Decoherence and Measurements”, Studies in History and Philosophy of Modern Physics 27: 239-277.

Casati, Giulio and Boris Chirikov (1995a), "Comment on 'Decoherence, Chaos, and the Second Law", Physical Review Letters 75: 349-350.

--- (1995b), “Quantum Chaos: Unexpected Complexity”, Physica D 86: 220-237.

Castagnino, Mario (2004), “The Classical-Statistical Limit of Quantum Mechanics”, Physica A 335: 511-517.

--- (2005), “Classical Limit of Non-Integrable Systems”, Brazilian Journal of Physics 35: 375-379.

--_ (2006), “The Equilibrium Limit of the Casati-Prosen Model”, Physics Letters A 357: 97-100.

Castagnino, Mario and Manuel Gadella (2006), “The Role of Self-Induced Decoherence in the Problem of the Classical Limit of Quantum Mechanics”, Foundations of Physics, forthcoming.

Castagnino, Mario, Manuel Gadella and Olimpia Lombardi (2005), “Time's Arrow and Irreversibility in Time-Asymmetric Quantum Mechanics”, International Studies in the Philosophy of Science 19: 223243.

Castagnino, Mario and Roberto Laura (2000a), "Functional Approach to Quantum Decoherence and the Classical Final Limit”, Physical Review A 62: 022107.

--- (2000b), "Functional Approach to Quantum Decoherence and the Classical Limit: the Mott and Cosmological Problems”, International Journal of Theoretical Physics 39: 1737-1765.

Castagnino, Mario, Roberto Laura and Olimpia Lombardi (2006), "Decoherence in Closed and Open Systems”, Los Alamos National Laboratory, arXiv:quant-ph/0601132.

Castagnino, Mario and Olimpia Lombardi (2003), "The Self-Induced Approach to Decoherence in Cosmology”, International Journal of Theoretical Physics 42: 1281-1299.

--- (2004), “Self-Induced Decoherence: A New Approach”, Studies in History and Philosophy of Modern Physics 35: 73-107.

--- (2005), “Decoherence Time in Self-Induced Decoherence”, Physical Review A 72: 012102.

--- (2006a), “The Classical Limit of Non-Integrable Quantum Systems, a Route to Quantum Chaos”, Chaos, Solitons and Fractals 28: 879-898.

--- (2006b), “Self-Induced Decoherence and the Classical Limit of Quantum Mechanics”, Philosophy of Science, forthcoming.

Castagnino, Mario and Adolfo Ordoñez (2004), “An Algebraic Formulation of Quantum Decoherence”, International Journal of Theoretical Physics 43: 695-717.

Daneri, A., A. Loinger, and G. M. Prosperi (1962), "Quantum Theory of Measurement and Ergodicity Conditions”, Nuclear Physics 33: 297-319. 
d'Espagnat, Bernard (1995), Veiled Reality. An Analysis of Present-Day Quantum Mechanical Concepts. Reading: Addison-Wesley.

Diosi, Lajos (1987), “A Universal Master Equation for the Gravitational Violation of Quantum Mechanics”, Physics Letters A 120: 377-381.

--_ (1989), “Models for Universal Reduction of Macroscopic Quantum Fluctuations”, Physical Review A 40: 1165-1174.

Elby, Andrew (1994), “The 'Decoherence’ Approach to the Measurement Problem in Quantum Mechanics”, Proceedings of the Philosophy of Science Association 1: 355-365.

Ford, George and Robert O'Connell (2001), “Decoherence without Dissipation”, Physics Letters A 286: 8790.

Frasca, Marco (2003), “General Theorems on Decoherence in the Thermodynamic Limit”, Physics Letters A 308: 135-139.

Healey, Richard (1995), “Dissipating the Quantum Measurement Problem”, Topoi 14: 55-65.

Joos, Erich, Dieter Zeh, Carl Kiefer, Domenico Giulini, Joachim Kupsch and Ion-Olimpiu Stamatescu (2003), Decoherence and the Appearance of a Classical World in Quantum Theory. Berlin: Springer-Verlag.

Lombardi, Olimpia (2002), "Determinism, Internalism and Objectivity”, in H. Atmanspacher and R. Bishop (eds.), Between Chance and Choice: Interdisciplinary Perspectives on Determinism. Exeter: ImprintAcademic.

Lombardi, Olimpia and Martín Labarca (2005), “The Ontological Autonomy of the Chemical World”, Foundations of Chemistry 7: 125-148.

Mackey, Michael (1989), “The Dynamic Origin of Increasing Entropy”, Reviews of Modern Physics 61: 9811015.

Milburn, Gerard (1991), “Intrinsic Decoherence in Quantum Mechanics”, Physical Review A 44: 5401-5406.

Omnes, Roland (2001), "Decoherence: An Irreversible Process”, Los Alamos National Laboratory, arXiv:quant-ph/0106006.

--_ (2002), "Decoherence, Irreversibility and the Selection by Decoherence of Quantum States with Definite Probabilities”, Physical Review A 65: 052119.

-_- (2005), “Results and Problems in Decoherence Theory”, Brazilian Journal of Physics 35: 207-210.

Paz, Juan Pablo and Wojciech Zurek (2002), "Environment-Induced Decoherence and the Transition from Quantum to Classical”, in A. J. Leggett (ed.), Lecture Notes in Physics, Vol. 587. Heidelberg-Berlin: Springer.

Pessoa Jr., Osvaldo (1998), “Can the Decoherence Approach Help to Solve the Measurement Problem?”, Synthese 113: 323-346.

Schlosshauer, Maximilian (2005), “The 'Self-Induced Decoherence' Approach: Strong Limitations on its Validity in a Simple Bath Model and on its General Physical Relevance", Physical Review A 72: 012109.

Sicardi Schifino, A. C., G. Abal, R. Siri, A. Romanelli and R. Donangelo (2003), "Intrinsic Decoherence and Irreversibility in the Quasiperiodic Kicked Rotor”, Los Alamos National Laborator, arXiv:quantph/0308162.

Stamp, Philip (1995), “Time, Decoherence and 'Reversible’ Measurements”, in S. Savitt (ed.), Time’s Arrow Today. Cambridge: Cambridge University Press. 
van Hove, Leon (1957), “The Approach to Equilibrium in Quantum Statistics: A Perturbation Treatment to General Order”, Physica 23: 441-480.

-_- (1959), “The Ergodic Behaviour of Quantum Many-Body Systems”, Physica 25: 268-276.

van Kampen, Nicolaas (1954) “Quantum Statistics of Irreversible Processes”, Physica 20: 603-622.

Zeh, Dieter (1970), “On the Interpretation of Measurement in Quantum Theory”, Foundations of Physics 1: 69-76.

--_ (1971), “On the Irreversibility of Time and Observation in Quantum Theory”, in B. d'Espagnat (ed.), Foundations of Quantum Mechanics. New York: Academic Press.

-_- (1973), “Toward a Quantum Theory of Observation”, Foundations of Physics 3: 109-116.

Zurek, Wojciech (1981), "Pointer Basis of Quantum Apparatus: Into What Mixtures does the Wave Packet Collapse?”, Physical Review D 24: 1516-1525.

--_ (1982), “Environment-Induced Superselection Rules”, Physical Review D 26:1862-1880.

-_- (1991), “Decoherence and the Transition from Quantum to Classical”, Physics Today 44: 36-44.

-_- (1994), "Preferred Sets of States, Predictability, Classicality and Environment-Induced Decoherence”, in J. J. Halliwell, J. Pérez-Mercader and W. H. Zurek (eds.), Physical Origins of Time Asymmetry. Cambridge: Cambridge University Press.

--- (1998), “Decoherence, Einselection, and the Existential Interpretation”, Los Alamos National Laboratory, arXiv:quant-ph/9805065.

--_ (2003), "Decoherence, Einselection, and the Quantum Origins of the Classical”, Reviews of Modern Physics 75: 715-776. 